\title{
Transient Analysis of Carbon Combustion in Stagnation Flow
}

\author{
F. MÉNDEZ C. TREVIÑo A. LIÑ̃́N
}

\begin{abstract}
The early history of a carbon particle is studied as it is suddenly injected into a hot oxidizing atmosphere. In this early phase, a high Reynolds number approach is appropriate. Five stages characterize the processes: (1) inert stage, (2) transition stage (ignition of the heterogeneous reaction), (3) heterogeneous diffusion controlled stage, (4) homogeneous ignition, and (5) overall diffusion-controlled stage. In this last stage, the surface temperature and the regression rate reach the final equilibrium values. Using correlations for the quasi-steady gas-phase response and Green's function for the solid phase, the evolution of the surface temperature as well the regression rate are obtained.
\end{abstract}

\section{NOMENCLATURE}

$C_{s} \quad$ specific heat of the solid

$C_{p} \quad$ specific heat at constant pressure of the gas

$D_{i} \quad$ diffusion coefficient of species $i$

$E_{h} \quad$ activation energy of the homogeneous reaction

$E_{\mathrm{I}}, E_{\mathrm{II}}$ activation energies of the heterogeneous reactions

$f \quad$ nondimensional stream function defined in Eq. 22

$\bar{J} \quad \sigma \bar{T}_{\infty}{ }^{3} \operatorname{Pr} \sqrt{R} / C_{p} \gamma \sqrt{\alpha \rho_{\infty} \mu_{\infty} U_{\infty}}$

$f$ nondimensional stream function defined in Eq. 22

$k$ preexponential factor of the homogeneous reaction

$\dot{m}$ mass flow rate at the carbon surface

$\dot{M}$ nondimensional mass flow rate at the surface defined in Eq. 27

Pr Prandtl number, $\operatorname{Pr}=\mu C_{p} / \lambda$

$Q$ heat of reaction of the homogeneous reaction per unit mass of $\mathrm{CO}$ consumed

$Q_{\mathrm{I}}, Q_{\mathrm{I}}$ heat of reaction of the heterogeneous reactions per unit mass of carbon consumed

$q_{E} \quad$ external heat flux

$r_{\mathrm{I}}, r_{\mathrm{II}}$ reaction rates of the heterogeneous reactions given by Eqs. 16 and 17

$R \quad$ particle radius

$\mathrm{Sc}_{i} \quad$ Schmidt number of species $i, \mathrm{Sc}_{i}=\mu /$ $\rho D_{i}$

$t \quad$ time

$\bar{T}_{0} \quad$ initial temperature of the particle

$\bar{T} \quad$ temperature

$U_{\infty} \quad$ free-stream velocity

$u, v \quad$ Cartesian components of the gas velocity on $x$ and $y$, respectively

$w_{i} \quad$ consumption rate of species $i$ per unit volume and time

$W_{i} \quad$ molecular weight of species $i$

$x, y \quad$ Cartesian coordinates with $x$ following the particle surface

$\dot{y} \quad$ regression rate of the carbon surface 
$\bar{Y}_{i} \quad$ mass concentration of species $i$ in the mixture

\section{Greek Symbols}

$\rho \quad$ gas density

$\gamma \quad$ nondimensional parameter, $\gamma=\left(\bar{T}_{\infty}-\right.$ $\left.\bar{T}_{0}\right) / \bar{T}_{\infty}$

$\eta \quad$ nondimensional normal coordinate defined in Eq. 22

$\xi \quad$ nondimensional coordinate introduced in Eq. 27

$\lambda$ coefficient of thermal conductivity

$\mu \quad$ coefficient of viscosity

$\nu_{i} \quad$ stoichiometric coefficient of species $i$ in the homogeneous reaction

$\psi \quad$ stream function

o nondimensional longitudinal coordinate introduced in Eq. 21

$\sigma \quad$ Stefan-Boltzmann constant

$\theta$ nondimensional temperature defined as $\theta=\left(\bar{T}_{\infty}-\bar{T}\right) /\left(\bar{T}_{\infty}-\bar{T}_{0}\right)$

$\tau$ nondimensional time defined in Eq. 29

\section{Subscripts}

$\begin{array}{ll}s & \text { solid } \\ w & \text { carbon surface } \\ 0 & \text { initial conditions for the carbon particle } \\ \infty & \text { free stream }\end{array}$

\section{INTRODUCTION}

The study of carbon combustion has received special attention in the literature in the past. Several flow geometries have been selected in order to study the interaction between fluid mechanics and homogeneous and heterogeneous kinetics. There are two basic trends regarding the fluid mechanical aspect of the flow considered. One is to assume that the carbon particle is in a quiescent oxidizer gas [1-8]. Ubhayakar [1] studied the quasi-steady burning and extinction of spherical carbon particles. Libby et al. [2, 3, 5, 6] analyzed the steady-state and transient combustion of carbon particles suddenly immersed in a hot oxidizing ambient. The effect of water vapor content was also evaluated [5]. Kassoy and Libby [6] used asymptotic methods for high Zeldovich numbers of the heterogeneous reaction for the study of the entire history of a carbon particle. They analyzed different regimes and compared them with the numerical solution of the governing equations. The critical conditions needed to obtain vigorous heterogeneous combustion have been obtained [8] and the particle lifetime was deduced for both subcritical and supercritical behavior. The other flow geometries selected are those of the boundary layer in stagnation and flat plate flows [9-14]. Matsui et al. [9] made an experiment to study the influence of the characteristics of the flow on the rate of consumption in a well-defined field. They measured the combustion rate of a carbon test specimen in a stagnation-point flow field, which can be described by the velocity gradient. In other experimental study, Matsui et al. [11] investigated the influence of humidity in the flow on the combustion rate. Adomeit et al. [12] made a theoretical study on the stagnation point flow configuration for comparison with the experimental data obtained by Matsui et al. [9, 11]. They concluded that the combustion behavior of solid carbon cannot be described by the limiting cases. Adomeit and Visser [13] made a very careful experiment, burning carbon in a stagnation-point flow. The dependence of the various physicochemical parameters on the combustion process was obtained.

The objective of the present work is to study the transient behavior of a carbon particle exposed to a stagnation-point boundary layer flow for large Reynolds number based on the particle radius, using asymptotic methods based on large Zeldovich numbers for both homogeneous and heterogeneous reactions.

\section{FORMULATION}

We consider a carbon particle of radius $R$ subjected to a convective flow of an oxidant (air). If we consider cases where the Reynolds number based on $R$ and the free stream is large-that is, the boundary layer thickness is very small compared with the particle radius--the boundary layer 
governing equations are given by

$$
\begin{aligned}
& \frac{\partial}{\partial x}(\rho u)+\frac{\partial}{\partial y}(\rho v)=0 \\
& \rho u \frac{\partial u}{\partial x}+\rho v \frac{\partial u}{\partial y}=\frac{\partial}{\partial y}\left(\mu \frac{\partial u}{\partial y}\right)+\rho_{\infty} \mu_{\infty} \frac{d u_{\infty}}{d x} \\
& \rho u C_{p} \frac{\partial \bar{T}}{\partial x}+\rho v C_{p} \frac{\partial \bar{T}}{\partial y}=\frac{\partial}{\partial y}\left(\lambda \frac{\partial \bar{T}}{\partial y}\right)+w Q \\
& \rho u \frac{\partial \bar{Y}_{i}}{\partial x}+\rho v \frac{\partial \bar{Y}_{i}}{\partial y}=\frac{\partial}{\partial y}\left(\rho D_{i} \frac{\partial \bar{Y}_{i}}{\partial y}\right)-w_{i} \\
& i=1, \cdots, N,
\end{aligned}
$$

where the notation is explained in the Nomenclature section. The boundary layer coordinates are $x$ and $y$, corresponding to the longitudinal coordinate following the surface of the particle and the transverse coordinate, respectively. The origin is located in the stagnation point of the flow. $w_{i}$ corresponds to the mass consumption rate of species $i$ per unit volume due to the homogeneous reaction $\left(\mathrm{CO}+\frac{1}{2} \mathrm{O}_{2} \rightarrow \mathrm{CO}_{2}\right)$. $w$ is the corresponding value for carbon monoxide $(\mathrm{CO})$, and $Q$ is the heat of reaction per unit mass of $\mathrm{CO}$ consumed. For the homogeneous reaction, $w$ is given by

$w=k \rho^{2}\left(\bar{Y}_{\mathrm{H}_{2} \mathrm{O}} \bar{Y}_{\mathrm{O}_{2}}\right)^{1 / 2} \bar{Y}_{\mathrm{CO}} \exp \left(-E_{h} / R \bar{T}\right)$

and

$w_{i}=-v_{i} w W_{i} / W_{\mathrm{CO}}$.

The boundary conditions associated to the freestream side for the equations given above are the following:

At $y \rightarrow \infty$ :

$u=u_{\infty}(x), \quad \bar{T}=\bar{T}_{\infty}$,

$\bar{Y}_{\mathrm{O}_{2}}=\bar{Y}_{\mathrm{O}_{2 \infty}}, \quad \bar{Y}_{\mathrm{CO}}=\bar{Y}_{\mathrm{CO}_{2}}=0$.

The mass and energy balance condition in the gassolid interface, considering no gas diffusion through the solid, are given by

$$
\rho D_{\mathrm{O}_{2}}\left(\frac{\partial \bar{Y}_{\mathrm{O}_{2}}}{\partial y}\right)_{w}-(\rho v)_{w} \bar{Y}_{\mathrm{O}_{2}}=\frac{r_{1}}{2} W_{\mathrm{O}_{2}}
$$

$$
\begin{aligned}
& \rho D_{\mathrm{CO}_{2}}\left(\frac{\partial \bar{Y}_{\mathrm{CO}_{2}}}{\partial y}\right)_{w}-(\rho v)_{w} \bar{Y}_{\mathrm{CO}_{2}}=r_{\mathrm{II}} W_{\mathrm{CO}_{2}} \\
& \rho D_{\mathrm{CO}}\left(\frac{\partial \bar{Y}_{\mathrm{CO}}}{\partial y}\right)_{w}-(\rho v)_{w} \bar{Y}_{\mathrm{CO}}=-\left(r_{\mathrm{I}}+r_{\mathrm{II}}\right) W_{\mathrm{CO}} \\
& \left(r_{\mathrm{I}}+r_{\mathrm{II}}\right) W_{C}=\dot{m}=(\rho v)_{w}=-\rho_{s} \dot{y} \\
& q_{E}+\lambda\left(\frac{\partial \bar{T}}{\partial y}\right)_{w}-\left(\lambda_{s} \frac{\partial \bar{T}_{s}}{\partial y}\right)_{w}+\dot{m} C_{s} \bar{T}_{w} \\
& \quad-\dot{m} C_{p} \bar{T}_{w}=-Q_{R}
\end{aligned}
$$

where $q_{E}$ denotes the overall external heat flux due to radiation and any other coming from an external heat device. $Q_{R}$ is the total heat of reaction per unit mass of carbon consumed, produced by the two heterogeneous reactions considered here,
(I) $\mathrm{C}+\frac{1}{2} \mathrm{O}_{2} \rightarrow \mathrm{CO}$
(II) $\mathrm{C}+\mathrm{CO}_{2} \rightarrow 2 \mathrm{CO}$,

and is given by

$Q_{R}=\left(r_{\mathrm{I}} Q_{\mathrm{I}}-r_{\mathrm{II}} Q_{\mathrm{II}}\right) W_{C}$.

$r_{\mathrm{I}}$ and $r_{\mathrm{II}}$ correspond to the reaction rates for both reactions, respectively, and are given by

$r_{\mathrm{I}}=k_{\mathrm{I}}\left(\bar{Y}_{\mathrm{O}_{2}}\right)_{w} \exp \left(-E_{\mathrm{l}} / R \bar{T}_{w}\right)$,

$r_{\mathrm{II}}=k_{\mathrm{II}}\left(\bar{Y}_{\mathrm{CO}_{2}}\right)_{w} \exp \left(-E_{\mathrm{II}} / R \bar{T}_{w}\right)$

The regression rate of the carbon surface due to the two heterogeneous reactions is denoted by $\dot{y}$. If we also assume that the penetration length of the heating front in the solid is also very small compared with the radius of the particle, that is, for times such as

$t \ll R^{2} \rho_{s} C_{s} / \lambda_{s}$,

the solid-phase energy equation is given by

$\rho_{s} C_{s} \frac{\partial \bar{T}_{s}}{\partial t}=\lambda_{s} \frac{\partial^{2} \bar{T}_{s}}{\partial y^{2}}$ 
with the initial and boundary conditions given by

$\bar{T}_{s}=\bar{T}_{0} \quad$ at $y \rightarrow-\infty \quad$ or $\quad t=0$.

\section{NONDIMENSIONAL GOVERNING EQUATIONS}

The free-stream longitudinal velocity can be expressed in the form

$u_{\infty}=\left(\alpha \sigma+\beta \sigma^{3}+\cdots\right) U_{\infty}$,

with

$\sigma=x / R$

which is valid up to terms of order $\sigma^{3}$.

Here $\alpha$ and $\beta$ are constants that depend on the body geometry for a sphere, $\alpha=3 / 2, \beta=1 / 4$. Equation 21 gives very good results for values of $\sigma$ $\leq 1$. Introducing the nondimensional variables

$\theta=\frac{\bar{T}_{\infty}-\bar{T}}{\bar{T}_{\infty}-\bar{T}_{0}}, \quad \eta=\left(\frac{\alpha U_{\infty}}{R \rho_{\infty} \mu_{\infty}}\right)^{1 / 2} \int_{0}^{y} \rho d y$,

$\psi=\left(U_{\infty} \mu_{\infty} \rho_{\infty} R \alpha\right)^{1 / 2} \sigma f(\eta, \sigma)$

$Y_{\mathrm{O}_{2}}=\frac{\bar{Y}_{\mathrm{O}_{2}}}{\bar{Y}_{\mathrm{O}_{2 \infty}}}, \quad Y_{\mathrm{CO}}=\frac{\bar{Y}_{\mathrm{CO}}}{\bar{Y}_{\mathrm{O}_{2 \infty}} a_{\mathrm{CO}}}$,

$Y_{\mathrm{CO}_{2}}=\frac{\bar{Y}_{\mathrm{CO}_{2}}}{\bar{Y}_{\mathrm{O}_{2 \infty}} a_{\mathrm{CO}_{2}}}$

with $a_{\mathrm{CO}}=2 W_{\mathrm{CO}} / W_{\mathrm{O}_{2}}$ and $a_{\mathrm{CO}_{2}}=2 W_{\mathrm{CO}_{2}} / W_{\mathrm{O}_{2}}$, the gas phase quasi-steady governing equations transform to

$$
\begin{aligned}
L\left(1, \frac{d f}{d \eta}, 0\right)= & \left(\frac{d f}{d \eta}\right)^{2} \\
& -\frac{\rho_{\infty}}{\rho}\left(1+\frac{4 \beta}{\alpha} \sigma^{2}+\frac{\beta^{2}}{\alpha^{2}} \sigma^{4}\right) \\
L(\mathrm{Pr}, \theta, 1)= & L\left(\mathrm{Sc}_{\mathrm{O}_{2}}, Y_{\mathrm{O}_{2}},-r\right) \\
= & L\left(\mathrm{Sc}_{\mathrm{CO}}, Y_{\mathrm{Co}},-r\right) \\
= & L\left(\mathrm{Sc}_{\mathrm{CO}_{2}}, Y_{\mathrm{CO}_{2}}, r\right)=0,
\end{aligned}
$$

where $L$ is a differential operator defined by

$$
\begin{aligned}
L(p, \chi, q)= & \frac{1}{p} \frac{\partial^{2} \chi}{\partial \eta^{2}}+f \frac{\partial \chi}{\partial \eta} \\
& -\sigma\left(\frac{\partial \chi}{\partial \sigma} \frac{\partial f}{\partial \eta}-\frac{\partial \chi}{\partial \eta} \frac{\partial f}{\partial \sigma}\right) \\
& +q \tilde{k} \rho \sqrt{Y_{\mathrm{O}_{2}}} Y_{\mathrm{Co}} \\
& \times \exp \left(-\frac{E / R \bar{T}_{\infty}}{1-\gamma \theta}\right)
\end{aligned}
$$

and

$r=\frac{\left(\bar{T}_{\infty}-\bar{T}_{0}\right) C_{p}}{Q \bar{Y}_{\mathrm{O}_{2 \infty}}}, \quad \gamma=\frac{\bar{T}_{\infty}-\bar{T}_{0}}{\bar{T}_{\infty}}$.

The nondimensional balance equations for the gas-solid interface are then given by

$$
\begin{aligned}
& \bar{q}_{E}-\left.\frac{\partial \theta}{\partial \eta}\right|_{w}+\left.\frac{\partial \theta_{s}}{\partial \xi}\right|_{w}=-\dot{M}\left(\bar{Q}_{\mathrm{I}}+\frac{\nu}{\gamma}-\nu \theta_{w}\right) \\
& +R_{\mathrm{II}}\left(\bar{Q}_{\mathrm{I}}+\bar{Q}_{\mathrm{II}}\right) \\
& \left.\frac{\partial Y_{\mathrm{CO}_{2}}}{\partial \eta}\right|_{w}=\dot{M} \mathrm{Le}_{\mathrm{CO}_{2}} Y_{\mathrm{CO}_{2}}+\frac{R_{\mathrm{II}} W_{\mathrm{CO}_{2}} L_{\mathrm{CO}_{2}}}{W_{\mathrm{C}} a_{\mathrm{CO}_{2}} \bar{Y}_{\mathrm{O}_{2 \infty}}} \\
& \left.\frac{\partial Y_{\mathrm{O}_{2}}}{\partial \eta}\right|_{w}=\dot{M} \operatorname{Le}_{\mathrm{O}_{2}}\left(Y_{\mathrm{O}_{2}}+\frac{W_{\mathrm{O}_{2}}}{2 W_{\mathrm{C}} \bar{Y}_{\mathrm{O}_{2 \infty}}}\right) \\
& -\frac{R_{\mathrm{II}} \mathrm{Le}_{\mathrm{O}_{2}} W_{\mathrm{O}_{2}}}{2 W_{\mathrm{C}} \bar{Y}_{\mathrm{O}_{2 \infty}}}
\end{aligned}
$$

$$
\begin{aligned}
&\left.\frac{\partial Y_{\mathrm{Co}}}{\partial \eta}\right|_{w}= \dot{M} \operatorname{Le}_{\mathrm{Co}}\left(Y_{\mathrm{CO}}-\frac{W_{\mathrm{CO}}}{W_{\mathrm{C}} a_{\mathrm{CO}} \bar{Y}_{\mathrm{O}_{2 \infty}}}\right) \\
&-\frac{R_{\mathrm{II}} \mathrm{Le}_{\mathrm{CO}} W_{\mathrm{Co}}}{W_{\mathrm{C}} a_{\mathrm{CO}} \bar{Y}_{\mathrm{O}_{2 \infty}}} \\
& \dot{M}=-\operatorname{Pr}\left(f+\sigma \frac{\partial f}{\partial \sigma}\right)_{w}, \quad \frac{\partial f}{\partial \eta}=0,
\end{aligned}
$$

where

$$
\nu=\left(C_{p}-C_{s}\right) / C_{p}
$$

$\bar{q}_{E}=q_{E} \frac{\operatorname{Pr} \sqrt{R}}{C_{p}\left(\bar{T}_{\infty}-\bar{T}_{0}\right)\left(\alpha \rho_{\infty} \mu_{\infty} U_{\infty}\right)^{1 / 2}}$

$\xi=\frac{C_{p}}{\operatorname{Pr} \lambda_{s}}\left(\frac{\alpha \rho_{\infty} \mu_{\infty} U_{\infty}}{R}\right)^{1 / 2}\left(y+\frac{1}{\rho_{s}} \int_{0}^{t} \dot{m} d t\right)$ 
$\bar{Q}_{\mathrm{I}}=\frac{Q_{\mathrm{I}}}{C_{p}\left(\bar{T}_{\infty}-\bar{T}_{0}\right)}, \quad \bar{Q}_{\mathrm{II}}=\frac{Q_{\mathrm{II}}}{C_{p}\left(\bar{T}_{\infty}-\bar{T}_{0}\right)}$

$\dot{M}=\dot{m} \operatorname{Pr}\left(R / \alpha \rho_{\infty} \mu_{\infty} U_{\infty}\right)^{1 / 2}$

$R_{i}=r_{i} W_{C} \operatorname{Pr}\left(R / \alpha \rho_{\infty} \mu_{\infty} U_{\infty}\right)^{1 / 2}$

$\tilde{k}=\frac{2 Q \bar{Y}_{\mathrm{H}_{2} \mathrm{O}}{ }^{1 / 2} \bar{Y}_{\mathrm{O}_{2 \infty}}{ }^{3 / 2} W_{\mathrm{Co}}}{W_{\mathrm{O}_{2}} C_{p} \alpha \gamma U_{\infty}}$.

Due to the regression rate of the surface of the carbon particle, the introduction of the new coordinate $\xi$ enables us to fix this surface at $\xi=0$. The energy balance equation in the solid therefore takes the form

$\frac{\partial \theta_{s}}{\partial \tau}+\dot{M}(\sigma) \frac{\partial \theta_{s}}{\partial \xi}=\frac{\partial^{2} \theta_{s}}{\partial \xi^{2}}$,

where the appropriate nondimensional time $\tau$ is given by

$\tau=\frac{\rho_{s} C_{s} \operatorname{Pr}^{2} \lambda_{s} t}{C_{p}^{2} \alpha \rho_{\infty} \mu_{\infty}}$.

The initial and boundary conditions are

$\theta_{s}=1 \quad$ at $\xi \rightarrow-\infty$ or $\tau=0$.

In the following section, the analysis is done in the stagnation-point region. The solution in this zone allows us to study further the transient behavior along the $x$ coordinate, due to the parabolicity of the boundary layer equations away from the stagnation-point region.

\section{STAGNATION-POINT ZONE $(\sigma \rightarrow$ 0)}

The governing equations for the stagnation-point zone are obtained easily from Eqs. 23, 26, and 28 in the limit $\sigma \rightarrow 0$. These are reduced to the following forms.

\section{Gas Phase}

$$
\begin{aligned}
f^{\prime \prime \prime}+f f^{\prime \prime}+1 & -\gamma \theta-f^{\prime 2}=0 \\
\frac{1}{\operatorname{Pr}} \theta^{\prime \prime}+f \theta^{\prime}= & -\kappa_{\rho} \sqrt{Y_{\mathrm{O}_{2}}} Y_{\mathrm{CO}} \\
& \times \exp \left(-\frac{E_{h}}{R \bar{T}_{\infty}(1-\gamma \theta)}\right)
\end{aligned}
$$

$$
\begin{aligned}
\frac{1}{\mathrm{Sc}_{i}} Y_{i}^{\prime \prime}+f Y_{i}^{\prime}= & \pm \frac{\bar{k}\left(\bar{T}_{\infty}-\bar{T}_{0}\right) C_{p} \rho}{Q \bar{Y}_{\mathrm{O}_{2 \infty}}} \sqrt{Y_{\mathrm{O}_{2}}} Y_{\mathrm{Co}} \\
& \times \exp \left(-\frac{E_{h}}{R T_{\infty}(1-\gamma \theta)}\right),
\end{aligned}
$$

where the minus sign corresponds to $Y_{\mathrm{CO}_{2}}$.

\section{Solid Phase}

$\frac{\partial \theta_{s}}{\partial \tau}+\dot{M} \frac{\partial \theta_{s}}{\partial \xi}=\frac{\partial^{2} \theta_{s}}{\partial \xi^{2}}$,

with the following boundary and initial conditions:

At $\eta \rightarrow \infty$ :

$f^{\prime}=1, \quad \theta=0, \quad Y_{\mathrm{O}_{2}}=1$,

$Y_{\mathrm{CO}}=Y_{\mathrm{CO}_{2}}=0$.

At $\eta=\xi=0$ :

$\bar{q}_{E}-\theta^{\prime}+\left(\frac{\partial \theta_{s}}{\partial \xi}\right)_{w}$

$$
=-\dot{M}\left(\bar{Q}_{\mathrm{I}}+\frac{\nu}{\gamma}-\nu \theta_{w}\right)+R_{\mathrm{II}}\left(\bar{Q}_{\mathrm{I}}+\bar{Q}_{\mathrm{II}}\right)
$$

$Y_{\mathrm{O}_{2}}^{\prime}=\dot{M} \operatorname{Le}_{\mathrm{O}_{2}}\left(Y_{\mathrm{O}_{2}}+\frac{W_{\mathrm{O}_{2}}}{2 W_{\mathrm{C}} \bar{Y}_{\mathrm{O}_{2 \infty}}}\right)$

$$
-\frac{R_{\mathrm{II}} \mathrm{Le}_{\mathrm{O}_{2}} W_{\mathrm{O}_{2}}}{2 W_{\mathrm{C}} \bar{Y}_{\mathrm{O}_{2 \infty}}}
$$

$$
Y_{\mathrm{CO}_{2}}^{\prime}=\dot{M} \mathrm{Le}_{\mathrm{CO}_{2}} Y_{\mathrm{CO}_{2}}+\frac{R_{\mathrm{II}} W_{\mathrm{CO}_{2}} \mathrm{Le}_{\mathrm{CO}_{2}}}{W_{\mathrm{C} a_{\mathrm{CO}_{2}} \bar{Y}_{\mathrm{O}_{2 \infty}}}}
$$

$Y_{\mathrm{Co}}^{\prime}=\dot{M} \mathrm{Le}_{\mathrm{CO}}\left(Y_{\mathrm{CO}}-\frac{W_{\mathrm{Co}}}{W_{\mathrm{C}} a_{\mathrm{CO}} \bar{Y}_{\mathrm{O}_{2 \infty}}}\right)$

$$
-\frac{R_{\mathrm{II}} \mathrm{Le}_{\mathrm{CO}} W_{\mathrm{CO}}}{W_{\mathrm{C}} a_{\mathrm{CO}} \bar{Y}_{\mathrm{O}_{2 \infty}}}
$$

$\dot{M}=-\operatorname{Pr} f, \quad f^{\prime}=0$,

As $\xi \rightarrow-\infty, \theta_{s}=1$ and for $\tau<0 \theta_{s}=1$ in the whole particle of carbon. Due to the moderately large value of the activation energy of reaction I, there are three different stages involved in the 
process assuming that the ambient temperature $\bar{T}_{\infty}$ is larger than that of the carbon particle at $t=0$, $\bar{T}_{0}$. There is first an inert or warm-up stage, where the temperature at the particle increases toward the value $\bar{T}_{\infty}$. Upon reaching a critical temperature at the surface, the heterogeneous chemical reaction $\left(\mathrm{C}+\frac{1}{2} \mathrm{O}_{2} \rightarrow \mathrm{CO}\right)$ becomes important and must be taken into account. In this transition stage, the reaction changes to be controlled by diffusion and the $\mathrm{O}_{2}$ is totally consumed at the surface of the carbon particle. In the diffusion-controlled stage, the temperature increases to reach asymptotically the equilibrium value. In this stage, carbon monoxide is generated at the surface of the particle and diffuses through the boundary layer. A combustible mixture $\left(\mathrm{CO}+\mathrm{O}_{2}\right)$ is produced, and the homogeneous chemical reaction can be switched on.

Because of its large activation energy, this homogeneous reaction will take place first in regions close to the wall. If the homogeneous reaction is ignited, the reaction front moves toward the equilibrium position. The $\mathrm{CO}_{2}$ generated by the reaction diffuses to the surface of the particle and to the free stream. The second heterogeneous reaction $\left(\mathrm{CO}_{2}+\mathrm{C} \rightarrow 2 \mathrm{CO}\right)$ can compete with the first one $\left(\mathrm{C}+\frac{1}{2} \mathrm{O}_{2} \rightarrow \mathrm{CO}\right)$.

In the following sections, employing activation energy asymptotic methods, the different stages are analyzed, making use of the fact that the activation energy of the first heterogeneous reaction is large. The homogeneous reaction, which can be expected to play a significant role only in the final stage, will be considered. Figure 1 shows schematically the surface nondimensional temperature history, where all these stages are indicated.

\section{INERT STAGE}

In the inert stage heat is transferred from the gas phase to the carbon particle. Due to this process, the temperature at the surface of the particle increases from $\bar{T}_{0}$ to values very close to a critical temperature $\bar{T}_{c}$ where the heterogeneous reaction has to be considered. If this critical temperature is larger than $\bar{T}_{\infty}$, then no ignition or combustion takes place in the carbon particle. If the value of $\bar{T}_{c}$ is lower than $\bar{T}_{\infty}$, but not very close to it, a rapid transition stage follows the inert stage, where the chemical reaction is important. Therefore, the inert stage ends as $\bar{T}_{w}$ approaches $\bar{T}_{c}$ in a first approximation. The governing equations valid for this inert stage are as follows.

\section{Gas Phase}

$$
\begin{aligned}
& f^{\prime \prime \prime}+f f^{\prime \prime}+1-\gamma \theta-f^{\prime 2}=0 \\
& \frac{1}{\operatorname{Pr}} \theta^{\prime \prime}+f \theta^{\prime}=0
\end{aligned}
$$

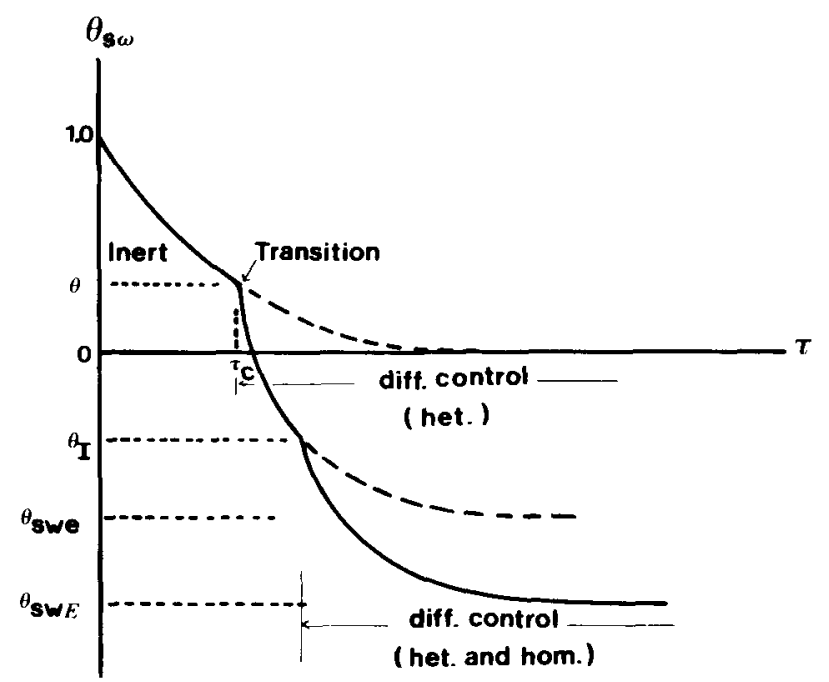

Fig. 1. Schematic surface nondimensional temperature history showing the different stages involved in the process. 
$Y_{\mathrm{O}_{2}}=1, \quad Y_{\mathrm{Co}}=Y_{\mathrm{CO}_{2}}=0$,

\section{Solid Phase}

$\frac{\partial \theta_{s}}{\partial \tau}=\frac{\partial^{2} \theta_{s}}{\partial \xi^{2}}$.

The boundary and initial conditions are as follows At $\eta \rightarrow \infty$ :

$f^{\prime}=1, \quad \theta=0$

At $\eta=\xi=0$ :

$\bar{q}_{E}-\theta^{\prime}+\frac{\partial \theta_{s}}{\partial \xi}=0, \quad f=f^{\prime}=0$

$$
\text { At } \xi \rightarrow-\infty \text { or } \tau<0 \text { : }
$$

$\theta_{s}=1$.

For the solid phase, the solution can be given in the form of an integral relationship between the temperature gradient and the temperature at the surface of the particle:

$\left.\frac{\partial \theta_{s}}{\partial \xi}\right|_{w}=\frac{1}{\sqrt{\pi}} \int_{0}^{\tau} \frac{d \theta_{s w}^{\prime}}{d \tau^{\prime}} \frac{d \tau^{\prime}}{\sqrt{\tau-\tau^{\prime}}}$.

Therefore, the energy-balance equation in the interface takes the form

$\theta_{w}^{\prime}-\bar{q}_{E}=\frac{1}{\sqrt{\pi}} \int_{0}^{\tau} \frac{d \theta_{s w}^{\prime}}{d \tau^{\prime}} \frac{d \tau^{\prime}}{\sqrt{\tau-\tau^{\prime}}}$.

Defining in the gas phase $\phi=\theta / \theta_{s w}$, the gas-phase equations transform to

$f^{\prime \prime \prime}+f f^{\prime \prime}+1-\bar{\gamma} \phi-f^{\prime 2}=0$,

$\frac{1}{\operatorname{Pr}} \phi^{\prime \prime}+f \phi^{\prime}=0$,

where $\bar{\gamma}=\gamma \theta_{s w}$. The boundary conditions are given by

At $\eta \rightarrow \infty$ :

$f^{\prime}=1, \quad \phi=0$

At $\eta=0$ :

$f=f^{\prime}=0, \quad \phi=1$.
The term $\bar{\gamma} \phi$ in Eq. 43 accounts for the reduction of the effect of the pressure gradient due to the increase in density when approaching the wall. Equations 43-45 have two parameters $\operatorname{Pr}$ and $\bar{\gamma}$. We can solve the equations using a perturbation technique with the incompressible solution, $\bar{\gamma}=$ 0 , as the unperturbed one. We assume a solution of the form

$f=f_{0}+\bar{\gamma} f_{1}+\cdots, \quad \phi=\phi_{0}+\bar{\gamma} \phi_{1}+\cdots$,

with $\bar{\gamma}$ taken to be a small number. Introducing relationship 46 into the boundary layer equations 43 and 44, we obtain the following set of equations:

$f_{0}^{\prime \prime \prime}+f_{0} f_{0}^{\prime \prime}+1-\left(f_{0}^{\prime}\right)^{2}=0$,

$\frac{1}{\operatorname{Pr}} \phi_{0}^{\prime \prime}+f_{0} \phi_{0}^{\prime}=0$

and

$f_{1}^{\prime \prime \prime}+f_{0} f_{1}^{\prime \prime}-2 f_{0}^{\prime} f_{1}^{\prime}+f_{0}^{\prime \prime} f_{1}=\phi_{0}$,

$\frac{1}{\operatorname{Pr}} \phi_{1}^{\prime \prime}+f_{0} \phi_{1}^{\prime}=-f_{1} \phi_{0}^{\prime}$.

The boundary conditions take the form

At $\eta \rightarrow \infty$ :

$f_{0}^{\prime}=1, \quad f_{1}^{\prime}=\phi_{0}=\phi_{1}=0$

At $\eta=0$ :

$f_{0}=f_{1}=f_{0}^{\prime}=f_{1}^{\prime}=\phi_{1}=0$,

$\phi_{0}=1$.

Equations 47 represent the very well known incompressible boundary layer flow [14]. From Eqs. 47 and 48 we need to know only the gradient at the wall

$\left.\frac{d \phi}{d \eta}\right|_{w} \approx \phi_{0}^{\prime}+\bar{\gamma} \phi_{1}^{\prime}$.

After numerical integration of Eqs. 47 and 48, we obtain

$\left.\frac{d \phi}{d \eta}\right|_{w} \approx-0.4958+0.0557 \bar{\gamma} \quad$ for $\bar{\gamma} \leq 0.75$. 
Introducing Eq. 51 into Eq. 42, this transforms to

$$
\begin{aligned}
& 0.4958 \theta_{s w}-0.0557 \gamma \theta_{s w}{ }^{2} \\
& +\bar{J}\left[1-\left(1-\gamma \theta_{s w}\right)^{4}\right]+\bar{q}_{\mathrm{Ex}} \\
& =-\frac{1}{\sqrt{\pi}} \int_{0}^{r} \frac{d \theta_{s w}^{\prime}}{d \tau^{\prime}} \frac{d \tau^{\prime}}{\sqrt{\tau-\tau^{\prime}}}
\end{aligned}
$$

where $\bar{q}_{\mathrm{Ex}}$ is the contribution to the heat flux at the interface from any other source except convection and radiation represented by the first terms in Eq. 52. The initial condition for Eq. 52 is that $\theta_{s w}(0)$ $=1$. For $\tau \rightarrow 0, \theta_{s w}$ from Eq. 52 behaves as

$$
\begin{aligned}
\theta_{s w}= & 1-\frac{2}{\sqrt{\pi}}\{0.4958-0.0557 \gamma \\
& \left.+\bar{J}\left[1-(1-\gamma)^{2}\right]+\bar{q}_{\mathrm{Ex}}\right\} \sqrt{\tau}+\cdots
\end{aligned}
$$

For large values of $\tau$, assuming a negligible chemical reaction, the temperature at the solid reaches asymptotically the value given by the solution to the algebraic equation

$$
\begin{aligned}
& 0.4958\left(\theta_{s w}\right)_{\mathrm{eq}}-0.0577\left(\theta_{s w}\right)_{\mathrm{eq}}{ }^{2} \\
& +\bar{J}\left\{1-\left[1-\gamma\left(\theta_{s w}\right)_{\mathrm{eq}}{ }^{4}\right]\right\}+\bar{q}_{\mathrm{Ex}}=0
\end{aligned}
$$

from the physical point of view, the root that behaves as

$\left(\theta_{s w}\right)_{\mathrm{eq}} \approx-\frac{q_{\mathrm{Ex}}}{0.4958+4 \bar{J} \gamma} \quad$ for $\bar{q}_{\mathrm{Ex}} \ll 1$

is the relevant one. In fact, the asymptotic behavior of $\theta_{s w}$ is given by

$\theta_{s w} \approx\left(\theta_{s w}\right)_{\text {eq }}+\frac{c}{\sqrt{\tau}}+\cdots \quad$ for $\tau \rightarrow \infty$

where

$$
\begin{aligned}
c= & \sqrt{\pi}\left(0.4958-0.154\left(\theta_{s w}\right)_{\mathrm{eq}}\right. \\
& \left.-\frac{4 \gamma \bar{J}\left\{1-\left[1-\gamma\left(\theta_{s w}\right)_{\mathrm{eq}}{ }^{4}\right]\right\}}{1+\gamma\left(\theta_{s w}\right)_{\mathrm{eq}}}\right)^{-1}
\end{aligned}
$$

The integral equation, Eq. 52, can be solved numerically. The evolution of $\theta_{s w}$ as a function of $\tau$ for the specific case of $\bar{q}_{\mathrm{Ex}}=\bar{J}=0$ is plotted in Fig. 2.

As the nondimensional temperature at the surface approaches the value $\theta_{s w}=\theta_{c}$, where $\theta_{c}$ is a critical nondimensional temperature to be defined

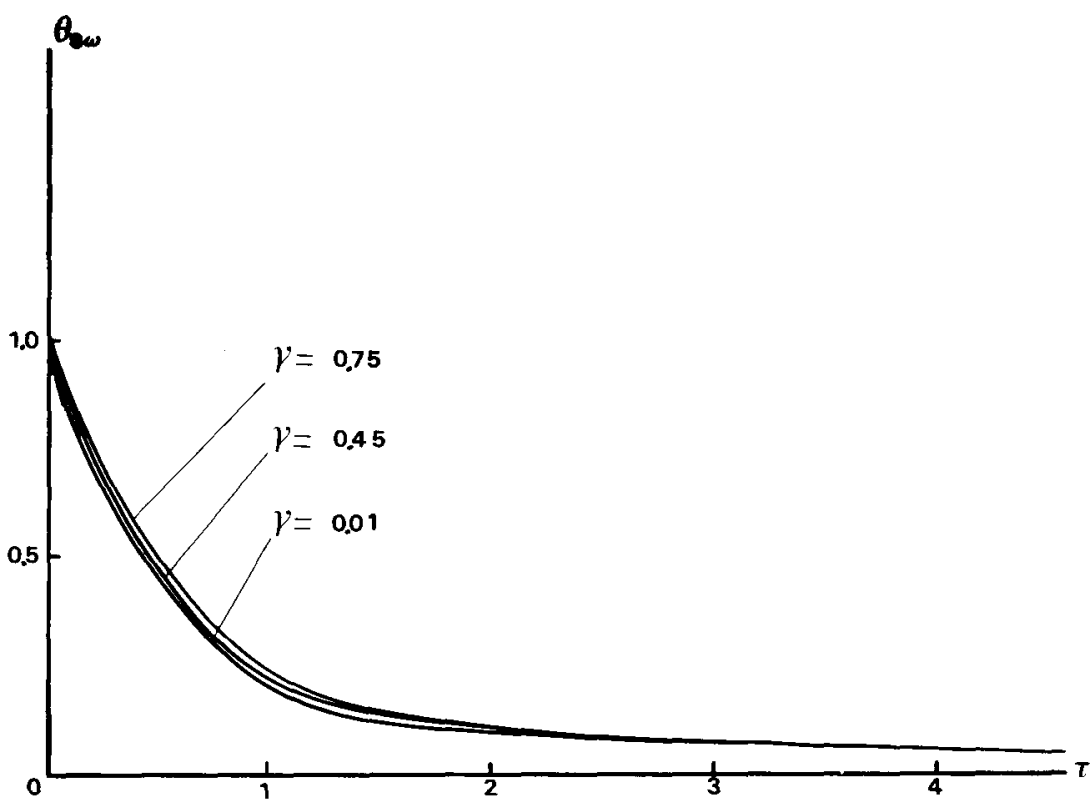

Fig. 2. Evolution of the nondimensional temperature at the surface, $\theta_{s w}$ as a function of $\gamma$. 
later, the chemical reaction has to be taken into account. This occurs at times $\tau$ close to $\tau_{c}$ defined by $\theta_{s w}\left(\tau_{c}\right)=\theta_{c}$. Here $\theta_{s w}$ can be given by

$$
\theta_{s w}=\theta_{c}+A\left(\theta_{c}, \bar{q}_{\mathrm{Ex}}, \bar{J}, \gamma\right)\left(\tau-\tau_{c}\right)+O\left(\tau-\tau_{c}\right)^{2} .
$$

The nondimensional heat generated by the heterogeneous chemical reaction is given by

$$
\begin{aligned}
\dot{M} & \left(\bar{Q}_{\mathrm{I}}+\frac{\nu}{\gamma}-\nu \theta_{s w}\right) \\
= & K_{\mathrm{I}} Y_{\mathrm{O}_{2 w}}\left(\bar{Q}_{\mathrm{I}}+\frac{\nu}{\gamma}-\theta_{s w} \nu\right) \\
& \times \exp \left[-\frac{E_{1}}{R \bar{T}_{\infty}\left(1-\gamma \theta_{s w}\right)}\right],
\end{aligned}
$$

where

$$
k_{\mathrm{I}}=\frac{k_{\mathrm{I}} W_{C} \operatorname{Pr} \sqrt{R}}{\sqrt{\alpha \rho_{\infty} \mu_{\infty}} U_{\infty}} \bar{Y}_{\mathrm{O}_{2 \infty}} \text {. }
$$

From Eqs. 58 and 52 we can obtain the critical value of the nondimensional temperature as

$\theta_{c} \approx \frac{1}{\gamma}\left(1-\frac{E_{1}}{R \bar{T}_{\infty} \ln \left(\bar{R}_{\mathrm{I}} \bar{Q}_{\mathrm{I}}\right)}\right)$.

As a function of $\theta_{c}$, the nondimensional heat of reaction is given as

$$
\begin{aligned}
\dot{M}\left(\bar{Q}_{\mathrm{I}}+\frac{\nu}{\gamma}-\nu \theta_{s w}\right) & \\
= & k_{\mathrm{I}} Y_{\mathbf{O}_{2 w}}\left(\bar{Q}_{\mathrm{I}}+\frac{\nu}{\gamma}-\nu \theta_{c}-\nu A\left(\tau-\tau_{c}\right)\right) \\
& \times \exp \left\{-E_{\mathrm{I}} /\left[R \bar{T}_{\infty}\left(1-\nu \theta_{c}\right)\right]\right\} \\
& \times \exp \frac{E_{\mathrm{I}} \gamma\left(\theta_{c}-\theta_{s w}\right)}{R \bar{T}_{\infty}\left(1-\gamma \theta_{c}\right)\left[1+\frac{\gamma\left(\theta_{c}-\theta_{s w}\right)}{\left(1-\gamma \theta_{c}\right)}\right]} .
\end{aligned}
$$

Owing to the fact that the activation energy for the heterogeneous reaction $\left(\mathrm{C}+\frac{1}{2} \mathrm{O}_{2} \rightarrow \mathrm{CO}\right)$ is large, the heat of reaction term is very large for $\theta_{s w}>\theta_{c}$ and very small for $\theta_{s w}<\theta_{c}$. Therefore, the inert solution ceases to be valid when the difference $\theta_{c}$ $-\theta_{s w} \approx R T_{0} / E_{\mathrm{I}}$ in the transition stage.

\section{TRANSITION STAGE}

In this stage we assume a nondimensional temperature to be given as a perturbation of the inertstage solution. This is given by

$\theta_{s}=\theta_{s \mathrm{I}}-\epsilon \psi_{s}+O\left(\epsilon^{2}\right)$,

where $\epsilon$ is a small number given by

$\epsilon=R \bar{T}_{c}^{2} / E_{\mathrm{I}}\left(\bar{T}_{\infty}-\bar{T}_{0}\right)$,

which is the inverse of the Zeldovich number. From Eq. 60 we know that the characteristic nondimensional time is of order $\epsilon$. Then we can introduce the appropriate time and length scales for this problem as

$\sigma=A\left(\tau-\tau_{c}\right) / \epsilon$

and

$Z=\sqrt{A / \epsilon} \xi$.

Introducing the variables $\psi_{s}, \sigma$, and $Z$, the energybalance equation for the gas-solid interface, given in Eq. 35, reduces to the lowest order

$\frac{\partial \psi_{s}}{\partial Z}=D \exp \left(\sigma+\psi_{s w}\right)$

where

$$
\begin{aligned}
D= & \frac{\bar{k}_{\mathrm{I}}}{\sqrt{A \epsilon}}\left(\bar{Q}_{\mathrm{I}}+\frac{\nu}{\gamma}-\nu \theta_{c}\right) \\
& \times \exp \left(-\frac{E_{\mathrm{I}}}{R \bar{T}_{\infty}\left(1-\gamma \theta_{c}\right)}\right) .
\end{aligned}
$$

The energy-balance equation in the solid transforms to

$$
\frac{\partial^{2} \psi_{s}}{\partial Z^{2}}=\frac{\partial \psi_{s}}{\partial \sigma},
$$

where the convective term is smaller than these terms. Using the same integral relationship be- 
tween the gradient and the value of $\psi_{s}$ at the surface, Eq. 65 takes the form

$\exp \left(x+\psi_{s w}\right)=\frac{1}{\sqrt{\pi}} \int_{-\infty}^{x} \frac{d \psi_{s w}^{\prime}}{d x^{\prime}} \frac{d x^{\prime}}{\sqrt{x-x^{\prime}}}$,

where $x$ is the new time scale defined as $x=\sigma+$ $\ln D$. The initial condition comes from matching with the inert stage

$\psi_{s} \rightarrow 0 \quad$ as $x \rightarrow-\infty$.

The solution to this integral equation can be found elsewhere [15]. There is a runaway at a value of $x_{I}$ $=-0.431$.

At the end of the transition stage, the chemical reaction rate is very large, thus consuming all the oxygen at the surface of the particle. At this time, the chemical reaction is limited by the diffusion of $\mathrm{O}_{2}$ to the surface.

\section{DIFFUSION-CONTROLLED STAGE}

To follow further the evolution of the temperature at the surface of the carbon particle with time, it is convenient to return to the original nondimensional variables $\theta, \tau$, and $\xi$. The governing equations are given by Eqs. $31-34$ without the gasphase reaction term. The initial condition is given by

$\theta=\theta_{s \mathrm{I}}\left(\xi, \tau_{c}\right) \quad$ at $\tau=\tau_{c}$.

Assuming a unit Lewis number for $\mathrm{O}_{2}$, we have that

$Y_{\mathrm{O}_{2}}=1-\phi \quad$ where $\phi=\theta / \theta_{s w}$.

From the $\mathrm{O}_{2}$ mass balance equation, Eq. 35, we obtain

$$
\begin{aligned}
\left(\frac{d Y_{\mathrm{O}_{2}}}{d \eta}\right)_{w} & =-\left.\frac{d \phi}{d \eta}\right|_{w}=\frac{\dot{M} W_{\mathrm{O}_{2}}}{2 W_{C} \bar{Y}_{\mathrm{O}_{2 \infty}}} \\
& =-\operatorname{Pr} f(0) \frac{W_{\mathrm{O}_{2}}}{2 W_{C} \bar{Y}_{\mathrm{O}_{2 \infty}}} .
\end{aligned}
$$

Therefore, Eq. 72 gives the boundary condition needed to solve the gas-phase governing equations. The nondimensional temperature gradient $d \phi / d \eta$ at the surface of the particle is plotted in Fig. 3 as a function of $\bar{\gamma}$. This gradient can be

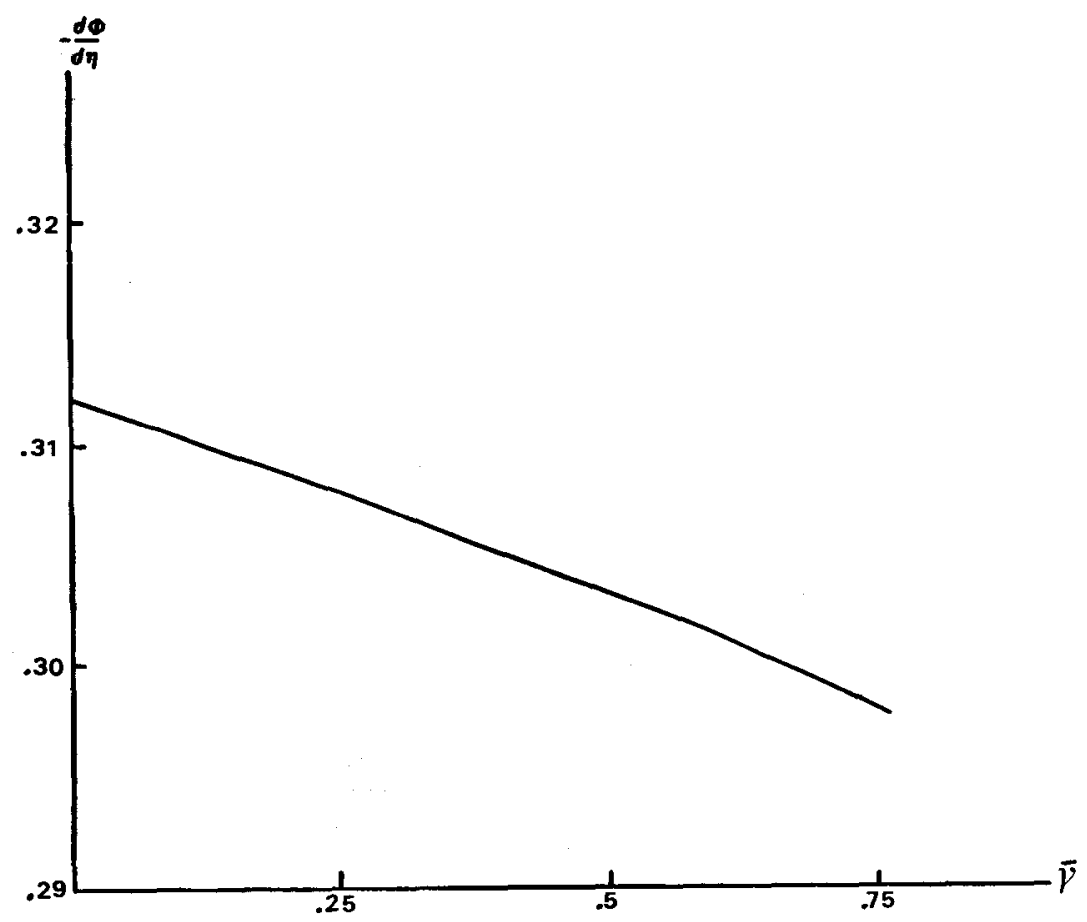

Fig. 3. Nondimensional heat flux at the surface, $(d \phi / d \eta)_{w}$ as a function of $\bar{\gamma}$. 
correlated very well with

$\left.\frac{d \phi}{d \eta}\right|_{w}=-0.4447+0.0489 \bar{\gamma}, \quad 0 \leq \bar{\gamma} \leq 0.75$.

(73)

Therefore,

$\dot{M}=\frac{2 W_{C}}{W_{\mathrm{O}_{2}}} \bar{Y}_{\mathrm{O}_{2 \infty}}\left(+0.4447-0.0489 \gamma \theta_{s w}\right)$.

The energy-balance equation at the solid-gas interface is given by

$$
\begin{aligned}
\left.\frac{\partial \theta_{s}}{\partial \xi}\right|_{w}= & -\dot{M}\left[\bar{Q}_{\mathrm{I}}+\frac{\nu}{\gamma}-\theta_{s w}\right. \\
& \left.\times\left(\frac{W_{\mathrm{O}_{2}}}{2 W_{C} \bar{Y}_{\mathrm{O}_{2 \infty}}}-\nu\right)\right]-\bar{q}_{E} .
\end{aligned}
$$

This is the required boundary condition for the solid-phase energy equation, Eq. 28, which can be considerably simplified if we use the following transformation:

$\theta(\xi, \tau)=W(\xi, \tau) \exp \left[\frac{\dot{M}}{2} \xi-\left(\frac{\dot{M}}{2}\right)^{2} \tau\right]$

The above change of variable is valid only for values of $\dot{M}$ constant; nevertheless, we can suppose that this condition is not too restrictive, because the effects introduced by the presence of the term $\gamma$ in Eq. 67 is weak and then it is a good approximation to take $\dot{M}$ as a constant as can be seen from Eq. 74 without the $\gamma$ term. Therefore the energy equation for the solid phase is reduced to

$$
\frac{\partial W}{\partial \tau}=\frac{\partial^{2} W}{\partial \xi^{2}},
$$

with the following conditions:

$$
\text { At } \tau=\tau_{c} \text { : }
$$

$W=\theta_{s I}\left(\xi, \tau_{c}\right) \exp \left[-\frac{\dot{M}}{2} \xi+\left(\frac{\dot{M}}{2}\right)^{2} \tau_{c}\right]$
At $\xi=0$ :

$$
\begin{aligned}
\left.\frac{\partial W}{\partial \tau}\right|_{w}= & -\dot{M} W\left[\frac{1}{2}+\left(\frac{W_{\mathrm{O}_{2}}}{2 W_{C} \bar{Y}_{\mathrm{O}_{2 \infty}}}-\nu\right)\right] \\
& -\dot{M} \exp \left[\left(\frac{\dot{M}}{2}\right)^{2} \tau\right]\left(\bar{Q}_{1}+\frac{\nu}{\gamma}\right)-\bar{q}_{E} .
\end{aligned}
$$

The problem defined by Eqs. 77-79 is linear, and it is possible to use the Green's function to solve it. After many manipulations we can arrive finally at the following expression for the solid temperature in this diffusion-controlled regime:

$$
\begin{aligned}
\theta(\xi, \tau)= & \exp \left[\frac{\dot{M}}{2} \xi-\left(\frac{\dot{M}}{2}\right)^{2} \tau\right] \\
& \times \int_{-\infty}^{0}\left(\exp \left[\frac{-\left(\xi-\xi^{\prime}\right)^{2}}{4\left(\tau-\tau_{c}\right)}\right]\right. \\
& +\exp \left[\frac{-\left(\xi+\xi^{\prime}\right)^{2}}{4\left(\tau-\tau_{c}\right)}\right]\left[2 \sqrt{\pi\left(\tau-\tau_{c}\right)}\right]^{-1} \\
& -A_{0} \exp \left[\left(\tau-\tau_{c}\right) A_{0}^{2}-A_{0}\left(\xi+\xi^{\prime}\right)\right] \\
& \left.\times \operatorname{erfc}\left(\frac{-\left(\xi+\xi^{\prime}\right)}{2 \sqrt{\tau-\tau_{c}}}+A_{0} \sqrt{\tau-\tau_{c}}\right)\right) \\
& \times \theta_{s 1}\left(\xi^{\prime}, \tau_{c}\right) \\
& \times \exp \left[\frac{\dot{M}}{2} \xi \xi^{\prime}+\left(\frac{\dot{M}}{2}\right)^{2} \tau_{c}\right] d \xi^{\prime} \\
& \left.\times B_{0} \exp \left[\left(\frac{\dot{M}}{2}\right)^{2} \tau\right]+C_{0}\right) d \tau^{\prime} \\
& +A_{0} \int_{0}^{\tau-\tau_{c}}\left(\left\{\exp \left[\frac{-\xi^{\prime 2}}{4\left(\tau-\tau^{\prime}\right)}\right]\right.\right. \\
& \times\left[\pi\left(\tau-\tau^{\prime}\right)\right]^{-1 / 2}-A_{0} \\
& \times \exp \left[A_{0}^{2}\left(\tau-\tau^{\prime}\right)-A_{0} \xi\right] \\
& \times \operatorname{erfc}\left(\frac{-\xi^{\prime}}{\left.\left.2 \sqrt{\tau-\tau^{\prime}}+A_{0} \sqrt{\tau-\tau^{\prime}}\right)\right\}}\right) \\
& \\
& \\
& \\
&
\end{aligned}
$$


where

$$
\begin{aligned}
& A_{0}=-\dot{M}\left[\frac{1}{2}+\left(\frac{W_{\mathrm{O}_{2}}}{2 W_{C} \bar{Y}_{\mathrm{O}_{2, \infty}}}-\nu\right)\right] \\
& B_{0}=-\dot{M}\left(\bar{Q}_{\mathrm{I}}+\nu / \gamma\right) \\
& C_{0}=-\bar{q}_{E}
\end{aligned}
$$

In Eq. 80 the value of $\theta_{s I}\left(\xi^{\prime}, \tau_{c}\right)$ can be obtained from the system of Eqs. 39-40 without the presence of the $\gamma$ term as was mentioned above, and is given by

$$
\begin{aligned}
\theta_{s I}\left(\xi^{\prime}, \tau_{c}\right)= & \operatorname{erfc}\left(\frac{-\xi^{\prime}}{2 \sqrt{\tau_{c}}}\right) \\
& +\exp \left(-A_{0} \xi^{\prime}+A_{0}^{2} \tau_{c}\right) \\
& \times \operatorname{erfc}\left(\frac{-\xi^{\prime}}{2 \sqrt{\tau_{c}}}+A_{0} \sqrt{\tau_{c}}\right)
\end{aligned}
$$

Given the complexity of Eq. 80, the most relevant characteristics of this equation appear to be

1. The heat flux in the carbon surface at initiation of the stage controlled by diffusion, that is, for times $\tau$ near $\tau_{c}$ and also

2. The final stage of this regime, which would be coupled with the equilibrium condition.

For the first, an asymptotic analysis shows that $\left.(\partial \theta / \partial \xi)\right|_{\xi=0}$ behaves according to the expression

$$
\begin{aligned}
\left.\frac{\partial \theta}{\partial \xi}\right|_{\xi=0} \approx & \frac{2 A_{0}^{2}}{M / 2-A_{0}}\left[\exp \left\{\left[\left(\frac{\dot{M}}{2}-A_{0}\right) \sqrt{\tau_{c}}\right]^{2}\right\}\right. \\
& \times \operatorname{erfc}\left(\frac{\dot{M}}{2}-A_{0}\right) \sqrt{\tau_{c}}-\exp \left[\left(A_{0} \sqrt{\tau_{c}}\right)^{2}\right] \\
& \times \operatorname{erfc}\left(A_{0} \sqrt{\tau_{c}}\right)-\exp \left[\left(\frac{\dot{M}}{2} \sqrt{\tau_{c}}\right)^{2}\right] \\
& \times \operatorname{erfc}\left(\frac{\dot{M}}{2} \sqrt{\tau_{c}}\right)+\frac{\dot{M} / 2-A_{0}}{\dot{M} / 2-2 A_{0}} \\
& \left.\times \exp \left(A_{0}{ }^{2} \tau_{c}\right)\right], \quad \text { as } \tau \rightarrow \tau_{c}
\end{aligned}
$$

and clearly it depends on $\dot{M}$ and $\tau_{c}$ fundamentally.

For the second characteristic, the contribution of the surface temperature comes from the second part of the right-hand side of Eq. 80, and it can be shown that for values of $\tau \rightarrow \infty$, the asymptotic solution is given by

$$
\begin{aligned}
\theta(0, \tau) \approx & \frac{2 A_{0} B_{0}}{\dot{M}}\left\{\operatorname{erfc}\left(\frac{\dot{M}}{2} \sqrt{\tau-\tau_{c}}\right)\right. \\
& -\frac{2 A_{0}}{\dot{M}}\left[1-\exp \left\{-\left[1-\left(\frac{2 A_{0}}{\dot{M}}\right)^{2}\right]\right.\right. \\
& \left.\left.\left.\times\left(\frac{\dot{M}}{2}\right)^{2}\left(\tau-\tau_{c}\right)\right\} \operatorname{erfc}\left(\tau-\tau_{c}\right)\right]\right\} \\
& \times\left[1-\left(\frac{2 A_{0}}{\dot{M}}\right)^{2}\right]^{-1} \quad \text { as } \tau \rightarrow \infty .
\end{aligned}
$$

The limit of which must coincide with the equilibrium value of $\theta$ to be obtained in the next section.

\section{EQUILIBRIUM STAGE WITHOUT HOMOGENEOUS REACTIONS}

As $t \rightarrow \infty$, the solution of the governing equation reaches asymptotically the equilibirum condition. The solid phase governing reduces to

$\dot{M}_{\mathrm{e}} \frac{d \theta_{s e}}{d \xi}=\frac{d^{2} \theta_{s e}}{d \xi^{2}}$,

with the boundary conditions given by

$\theta_{\text {se }}=\theta_{\text {swe }} \quad$ at $\xi=0$

and

$\theta_{s e} \rightarrow 1 \quad$ at $\xi \rightarrow-\infty$.

The solution of these equations is

$\theta_{s e}=1+\left(\theta_{s w e}-1\right) \exp \left(\dot{M}_{e} \xi\right)$

or

$\left.\frac{d \theta_{s e}}{d \xi}\right|_{w}=\left(\theta_{s e}-1\right) \dot{M}_{e}$.

Introduction of this relationship into the interface balance equations gives

$\theta_{s w e}\left(\frac{W_{\mathrm{O}_{2}}}{2 W_{\mathrm{C}} \bar{Y}_{\mathrm{O}_{2 \infty}}}-\nu+1\right)=\left(1-\bar{Q}_{\mathrm{I}}-\frac{\nu}{\gamma}\right)-\frac{\bar{q}_{E}}{\dot{M}_{e}}$ 
where

$\dot{M}_{e}=\left(+0.4447-0.0489 \gamma \theta_{s w e}\right) \frac{2 W_{\mathrm{C}} \bar{Y}_{\mathrm{O}_{2 \infty}}}{W_{\mathrm{O}_{2}}}$

and

$\bar{q}_{E}=\bar{J}\left[1-\left(1-\gamma \theta_{s w e}\right)^{4}\right]+\bar{q}_{\mathrm{Ex}}$.

The final equilibrium temperature can be obtained after solving the fourth-order algebraic equation for $\theta_{\text {swe }}$ resulting from Eqs. 88-90.

\section{INFLUENCE OF THE HOMOGENEOUS REACTION}

In the previous sections the different stages for the carbon combustion were analyzed assuming the gas phase to be frozen. However, as the temperature on the particle surface increases in the diffusion-controlled stage, the gas-phase reaction

$\mathrm{CO}+\frac{1}{2} \mathrm{O}_{2} \rightarrow \mathrm{CO}_{2}$

can be switched on because of the high sensitivity of the reaction rate on the temperature.

In a first approximation, the gas-phase chemical reaction has to be considered when the surface temperature reaches a critical value $\tilde{T}_{I}$ to be defined later. In practical cases, $\bar{T}_{\mathrm{I}}$ can be assumed to be larger than the ambient temperature, $\bar{T}_{\mathrm{I}}>$ $\bar{T}_{\infty}$. Thus, we can introduce the variable $\psi$ of order unity, which takes into account the effects of the gas-phase reaction. In this case, the nondimensional temperature profile close to the carbon surface is given by

$\theta=\theta_{1}-\left(0.4447 \theta_{1}-0.0489 \gamma \theta_{\mathrm{I}}^{2}\right) \eta-\epsilon \psi$,

$$
\text { as } \eta \rightarrow 0 \text {. }
$$

Here $\epsilon$ represents the inverse of the gas-phase Zeldovich number given by

$\epsilon=\frac{R \bar{T}_{\infty}\left(1-\gamma \theta_{\mathrm{I}}\right)^{2}}{\gamma E_{h}} \ll 1$.

We introduce also the inner stretched coordinate $X$, where the gas-phase chemical reaction takes place, as

$X=\frac{0.4447\left|\theta_{\mathrm{I}}\right|-0.0489 \gamma \theta_{\mathrm{I}}^{2}}{\epsilon} \eta$.

To the lowest order of $\epsilon$, the gas-phase energy equation takes the form

$\frac{d^{2} \psi}{d X^{2}}=-\Delta(X-\psi)^{1 / 2} \exp [\psi-X)$,

where $\Delta$ represents the relevant Damköhler number of order unity and is defined as

$\Delta=\frac{\tilde{k} \operatorname{Pr} \epsilon^{3 / 2} \rho_{\mathrm{I}} Y_{\mathrm{CO}, W} \exp \left[-E_{h} / R \bar{T}_{\infty}\left(1-\gamma \theta_{\mathrm{I}}\right)\right]}{\left(0.4447 \theta_{\mathrm{I}}\right)^{2} \sqrt{\left|\theta_{\mathrm{I}}\right|}\left[1-0.1101\left|\theta_{\mathrm{I}}\right|\right]^{2}}$.

(95)

Here, $Y_{\mathrm{Co}, W}$ represents the surface concentration of $\mathrm{CO}$, which is given by

$$
Y_{\mathrm{CO}, W}=\frac{1}{\left(2 W_{\mathrm{C}} / W_{\mathrm{O}_{2}}\right) \bar{Y}_{\mathrm{O}_{2 \infty}}-(d \phi / d \eta)_{w}} .
$$

Equation 94 has to be solved with the boundary conditions

$\psi(0)=0, \quad \frac{d \psi}{d X}(\infty)=0$.

The first is due to the fact that the characteristic time in the solid is much larger than in the gas. Therefore, in the homogeneous ignition process, the solid temperature is assumed to be constant. The second boundary condition is obtained from matching with the outer nonreactive zone. A first integration of Eqs. 94 and 97 gives

$\left.\frac{d \psi}{d X}\right|_{w}=-1 \pm \sqrt{1-2 \Gamma(3 / 2) \Delta}$,

where $\Gamma(\mathrm{n})$ represents the gamma function. The critical Damköhler number $\Delta$ is then

$\Delta_{\mathrm{I}}=1 / 2 \Gamma(3 / 2)$.

From Eq. 95 we can obtain the value of the critical temperature, $\bar{T}_{\mathrm{I}}$.

This ignition process takes place instantaneously when using the time scale of the solid. The 
gas-phase reaction front moves to the equilibrium position. The temperature at the surface of the carbon particle increases further to reach asymptotically the final equilibrium regime (when considering both homogeneous and heterogeneous reactions). In this final regime, from Eqs. 32 and 33 , using unit Lewis numbers for $\mathrm{O}_{2}$ and $\mathrm{CO}$, we obtain the following coupling relationships in the gas phase:

$Y_{\mathrm{O}_{2}}-Y_{\mathrm{CO}}=1-\phi$

$\theta-\bar{Q} Y_{\mathrm{O}_{2}}=-\bar{Q}+\left(\theta_{s w E}+\bar{Q}\right) \phi$,

where

$\bar{Q}=Q Y_{\mathrm{O}_{2}} / C_{p}\left(\bar{T}-\bar{T}_{0}\right)$

and $\theta_{s w E}$ is the equilibrium nondimensional temperature at the surface. In the above equations we have assumed zero oxygen and carbon monoxide concentrations in the carbon surface. In this case, the gas-phase flame sits at the carbon surface. In most practical cases, this is the appropriate regime, due to the fact that the heterogeneous reaction II (Eq. 14) is very slow in comparison with reaction I (Eq. 13). From Eqs. 100 and 101 we obtain

$\left.\frac{d Y_{\mathrm{O}_{2}}}{d \eta}\right|_{+}-\left.\frac{d Y_{\mathrm{CO}}}{d \eta}\right|_{+}=-\left.\frac{d \phi}{d \eta}\right|_{w}$

$\left.\frac{d Y_{\mathrm{O}_{2}}}{d \eta}\right|_{-}-\left.\frac{d Y_{\mathrm{co}}}{d \eta}\right|_{-}=-\left.\frac{d \phi}{d \eta}\right|_{w}$

$\left.\frac{d \theta}{d \eta}\right|_{-}-\left.\bar{Q} \frac{d Y_{\mathrm{O}_{2}}}{d \eta}\right|_{-}=\left.\left(\theta_{s w E}+\bar{Q}\right) \frac{d \phi}{d \eta}\right|_{w}$,

where the sign corresponds to the position relative to the gas-phase flame. The positive sign corresponds to the free-stream side and the negative sign to the carbon side of the interface. At the carbon surface, from Eqs. 35 we obtain

$\left.\frac{d Y_{\mathrm{O}_{2}}}{d \eta}\right|_{w}=\frac{\dot{M}_{E} W_{\mathrm{O}_{2}}}{2 W_{c} \bar{Y}_{\mathrm{O}_{2 \infty}}}=-\left.\frac{d Y_{\mathrm{CO}}}{d \eta}\right|_{w}$.

Due to the fact that in this case the conditions at the surface coincide with the conditions at the lower side of the gas-phase interface, $d Y_{i} /\left.d \eta\right|_{-}$ $=d Y_{i} /\left.d \eta\right|_{w}$ and $d \theta /\left.d \eta\right|_{-}=d \theta /\left.d \eta\right|_{w}$, then from Eqs. 103 and 105 we obtain the nondimensional mass flow rate at the surface,

$\dot{M}_{E}=-\left.\frac{W_{\mathrm{C}} \bar{Y}_{\mathrm{O}_{2}}}{W_{\mathrm{O}_{2}}} \frac{d \phi}{d \eta}\right|_{w}=-\operatorname{Pr} f(0)$.

Equation 106 gives the additional boundary condition needed to solve the system of Eqs. 43 and 44. Up to the first order in $\bar{\gamma}$, the solution to these equations is given by

$\left.\frac{d \phi}{d \eta}\right|_{w}=-0.4717+0.0252 \bar{\gamma}$.

The nondimensional gradient of temperature at the carbon surface is given then by

$\left.\frac{d \theta}{d \eta}\right|_{w}=\left.\left(\theta_{s w E}+\frac{1}{2} \bar{Q}\right) \frac{d \phi}{d \eta}\right|_{w}$.

From Eq. 87, the nondimensional heat transfer in the solid at the surface is given by

$\left.\frac{d \theta_{s E}}{d \xi}\right|_{w}=\left(\theta_{s w E}-1\right) \dot{M}_{E}$

Finally, from the energy balance at the surface we obtain the relationship needed to obtain the equilibrium temperature as

$$
\begin{aligned}
& \theta_{s w E}\left(\frac{W_{\mathrm{O}_{2}}}{W_{\mathrm{C}} \bar{Y}_{\mathrm{O}_{2 \infty}}}+1-\nu\right) \\
& \quad=1-\bar{Q}_{\mathrm{I}}-\frac{\nu}{\gamma} \frac{W_{\mathrm{O}_{2}}}{W_{\mathrm{C}} Y_{\mathrm{O}_{2 \infty}}} \frac{1}{2} \bar{Q}-\frac{\bar{q}_{E}}{\dot{M}_{E}},
\end{aligned}
$$

where

$\bar{q}_{E}=\bar{J}\left[1-\left(1-\gamma \theta_{s w E}\right)^{4}\right]+\bar{q}_{\mathrm{Ex}}$

$\dot{M}_{E}=\frac{W_{\mathrm{C}} \bar{Y}_{\mathrm{O}_{2 \infty}}}{W_{\mathrm{O}_{2}}}\left[0.4717-0.0252 \gamma \theta_{s w E}\right]$.

The transient process, after the ignition of the homogeneous reaction leading to the final equilibrium stage, can be analyzed using the same results obtained in the previous section, but with the appropriate mass flow rate $\dot{M}_{E}$ and initial conditions. 


\section{FINAL REMARKS}

An analysis has been performed on the transient combustion of a carbon particle exposed to a hightemperature oxidizing stagnation-point flow. This type of configuration represents the early stages of carbon particles injected into a hot oxidizing environment. In the analysis it was assumed that the thermal penetration length in the solid is much smaller than the particle radius. Asymptotic methods based on large Zeldovich numbers of both heterogeneous and homogeneous reactions were employed to analyze the different combustion stages. In the first phase of the process (frozen homogeneous reaction), there are three different stages, assuming the temperature of the oxidizer flow is higher than that of the carbon particle at the time of injection. The first or inert stage corresponds to a warm-up process, where the surface temperature increases. As the surface temperature reaches a first critical value, the heterogeneous reaction switches on. In this second transition stage, the heterogeneous reaction changes from kinetic to diffusion control and the oxygen is totally consumed at the surface of the carbon particle. In the third or diffusion-controlled stage, the temperature reaches asymptotically the equilibrium value (with frozen homogeneous reaction) with a constant regression rate. Using appropriate correlations for the gas phase and a Green's function for the solid phase, the evolution of surface temperature and regression rates are obtained. However, if the second critical temperature is lower than the equilibrium value, the gasphase reaction is important and a gaseous flame is generated in regions close to the wall. Here all the carbon monoxide is consumed and carbon dioxide is generated. A new regression rate is imposed on the carbon particle and the temperature at the surface reaches asymptotically the final equilibrium regime.

F. M. thanks the Ministerio de Educación y Ciencia of Spain, through the Scientific and Cultural Exchange Program between Mexico and Spain, for supporting a three-month stay at UPM in Madrid. C. T. thanks the Alexander von Humboldt Stiftung of Germany for support during his stays in Aachen and Madrid.

\section{REFERENCES}

Ubhayakar, S. K., Combust. Flame 26:23 (1976).

Libby, P. A., and Blake, T. R., Combust. Flame 36:139 (1979).

Libby, P. A., Combust. Flame 38:285 (1980)

Matalon, M., Combust. Sci. Technol. 24:115 (1980).

Libby, P. A., and Blake, T. R., Combust. Flame 41:123 (1981).

Kassoy, D. R., and Libby, P. A., Combust. Flame 48:287 (1982).

Matalon, M., SIAM J. Appl. Math. $42: 787$ (1982)

Trevino, C., Higuera, F., and Liñán, A., Critical conditions for carbon combustion, 21st Symposium (International) on Combustion, The Combustion Institute, 1987.

Matsui, K., Koyama, A., and Vehara, K., Combust. Flame 25:57 (1975).

Tsuji, H., and Matsui, K., Combust. Flame 26:283 (1976).

Matsui, K., Tsuji, H., and Makino, A., Combust. Flame 50:107 (1983).

Adomeit, G., Hocks, W., and Hendriksen, K., Combust. Flame 59:107 (1985).

Adomeit, G., and Visser, W., 20th Symposium (International) on Combustion, The Combustion Institute, 1985. Schlichting, H., Boundary Layer Theory, McGrawHill, New York, 1978.

Liñán, A., and Williams, F., Combust. Sci. Technol. 3:91 (1971). 